\title{
Nonparametric Methods for Doubly Truncated Data
}

\author{
Bradley Efron and Vahé Petrosian
}

\begin{abstract}
Truncated data plays an important role in the statistical analysis of astronomical observations as well as in survival analysis. The motivating example for this paper concerns a set of measurements on quasars in which there is double truncation. That is, the quasars are only observed if their luminosity occurs within a certain finite interval, bounded at both ends, with the interval varying for different observations. Nonparametric methods for the testing and estimation of doubly truncated data are developed. These methods extend some known techniques for data that is only truncated on one side, in particular Lynden-Bell's estimator and the truncated version of Kendall's tau statistic. However the kind of hazard function arguments that underlie the one-sided methods fail for two-sided truncation. Bootstrap and Markov Chain Monte Carlo techniques are used here in their place. Finally, we apply these techniques to the quasar data, answering a question about their long-term luminosity evolution.
\end{abstract}

Key Words: tau test, Lynden-Bell estimator, bootstrap, hypothesis test, Markov chain Monte Carlo, quasars, luminosity evolution, self-consistency.

Acknowledgement We are grateful to Susan Holmes, Persi Diaconis, and Duncan Murdach for guidance on the MCMC methodology. 


\section{Introduction}

Figure 1 shows an example of doubly truncated astronomical data. The plotted points are redshifts $z_{i}$ and $\log$ luminosities $y_{i}$ for $\mathrm{n}=210$ quasars, as explained in Section 6. Due to experimental constraints the distribution of each $y_{i}$ is truncated to a known interval $R_{i}$ depending on $z_{i}$. Truncation means that we would not have learned of $y_{i}$ 's existence if it fell outside of region $R_{i}$. Many experimental situations lead to truncated data, see for example McLauren et al. (1991). The double truncation seen in Figure 1, where $y_{i}$ goes undetected if it is either too small or too large, is less common than one-sided truncation. The quasar data has still another kind of truncation, of the redshift values $z_{i}$, but that will not affect our discussion.

We will describe nonparametric methods that answer to two related questions concerning truncated data:

Question 1 How can we test whether or not the $y_{i}$ 's are independent of the $z_{i}$ 's?

Question 2 Assuming independence, how can we estimate the marginal distribution of the $y_{i}$ 's?

The answers apply to all forms of truncation, including the double truncation of Figure 1 . In fact some of our methods apply to all forms of data censoring and truncation, as mentioned at the end of Section 5.

Question 1 turns out to be crucial for the scientific question posed by the quasars. A positive dependence of luminosity on redshift would mean that earlier quasars were intrinsically brighter, or in other words that quasars have been evolving toward a dimmer state as the universe ages. Figure 1 certainly seems to show a strong positive dependence between redshift and luminosity, but that appearance is forced on us by the truncation limits, which increase sharply from left to right. The tests described in Sections 2, 3, 5, and 6 will show that a statistically significant positive relationship remains after accounting for truncation, 


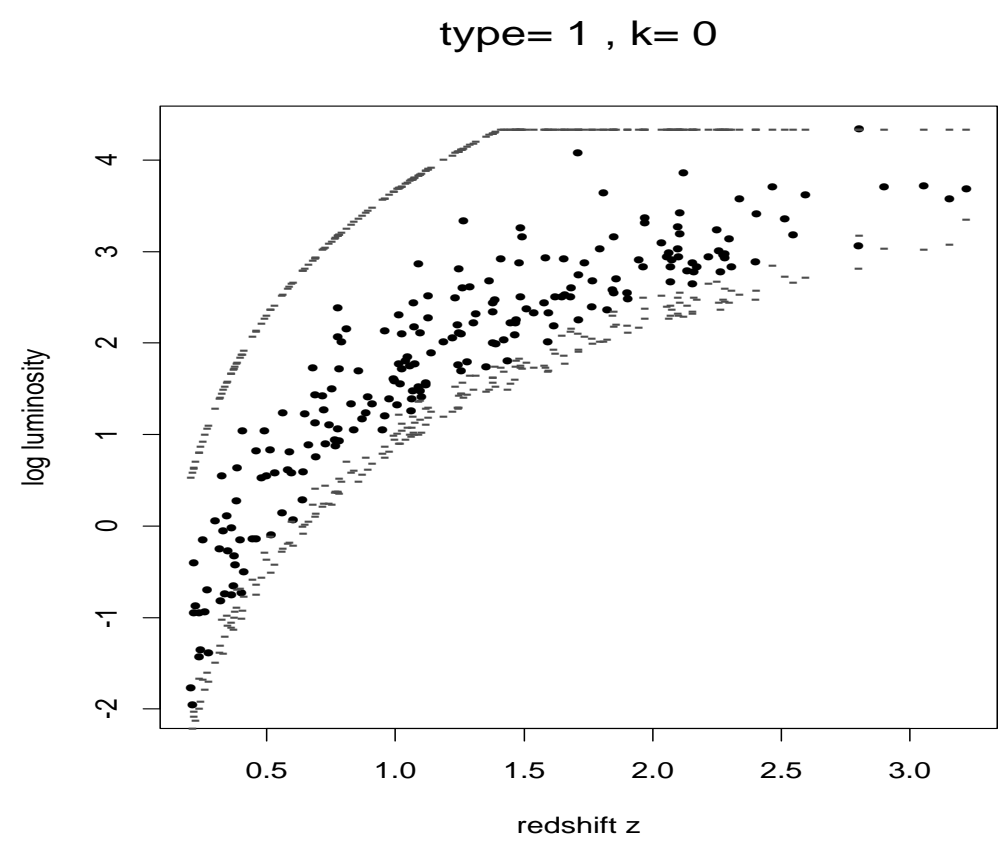

Figure 1: Doubly truncated data; points represent redshifts and luminosities for 210 quasars, as described in Section 6; luminosity subject to lower and upper truncation as indicated by dashes (lower truncation limits shifted down .25 for clarity.) Is luminosity correlated with redshift? 
though of much smaller magnitude than the figure suggests.

Turnbull's important 1976 paper answers Question 2 for arbitrary patterns of data truncation and censorship. Turnbull uses a self-consistency algorithm to calculate the nonparametric maximum likelihood estimate (MLE) of the $y$ distribution. His equations take on interesting forms for doubly truncated data, as discussed in Section 4.

Both Question 1 and Question 2 have simple closed-form solutions in the one-sided case, where the truncation regions $R_{i}$ extend upwards to infinity. Tsai (1990) and also Efron and Petrosian $(1992,1994)$ answer Question 1 using a version of Kendall's tau statistic appropriate to one-sided truncation. Lynden-Bell's (1971) answer to Question 2 in the onesided case is closely related to the Kaplan-Meier estimate for censored data, see Efron and Petrosian (1994).

Sections 2-5 extend these ideas to two-sided truncation (the extensions applying with little change to any pattern of multiple truncation.) The two-sided case is less tractable for both questions. The testing problem of Question 1 is particularly challenging computationally, and raises issues concerning the relationship of bootstrap methods to Markov Chain Monte Carlo (MCMC).

Lynden-Bell's method is completely nonparametric, as is the tau test, but it can be quite inefficient in some circumstances. Section 4 also discusses more efficient parametric estimates based on special exponential families, as in Efron (1996) and Efron and Tibshirani (1996).

We return to the quasar data in Section 6. The testing and estimation methods developed in Sections 2-5 are used there to answer Questions 1 and 2.

\section{Permutation Tests for Independence}

Doubly truncated data of the kind shown in figure 1 can be described as follows: the 
observed data consists of $n$ pairs $\left(z_{i}, y_{i}\right), y_{i}$ a real-valued response and $z_{i}$ in covariate, with observation $y_{i}$ restricted to a known region $R_{i}=\left[u_{i}, v_{i}\right]$,

$$
\text { data }=\left\{\left(z_{i}, y_{i}\right) \quad \text { with } \quad y_{i} \in R_{i}=\left[u_{i}, v_{i}\right] \text { for } i=1,2, \ldots, n\right\}
$$

The $n$ quadruplets $\left(z_{i}, y_{i}, u_{i}, v_{i}\right)$ are observed independently of one another. The regions $R_{i}$ can depend on $z_{i}$, as in figure 1 , and the $z_{i}$ values themselves can be subject to observational truncation.

Question 1 concerns testing the independence hypothesis $H_{o}$ : that if we could observe the $y_{i}$ 's without truncation they would be independent and identically distributed (i.i.d.) according to some common density function $f(y)$. Because of truncation, $H_{o}$ says that $y_{i}$ has the conditional density of $f(y)$ restricted to $R_{i}$,

$$
\begin{aligned}
H_{o}: \quad f\left(y_{i} \mid R_{i}\right) & =f\left(y_{i}\right) / F_{i} \text { for } y_{i} \in R_{i} \\
& =0 \text { for } y_{i} \notin R_{i}
\end{aligned}
$$

independently for $i=1,2, \ldots, n$. Here

$$
F_{i}=\int_{u_{i}}^{v_{i}} f(y) d y
$$

Question 2 asks us to estimate $f(y)$ assuming that $H_{o}$ is true.

This section discusses permutation tests of the independence hypothesis $H_{o}$. Table 1 and Figure 2 show an artificial example of truncated data involving $n=7$ points that will be helpful in carrying out the discussion.

A permutation of $\mathbf{y}=\left(y_{1}, y_{2}, \ldots, y_{n}\right)$, say $\mathbf{y}^{*}=\left(y_{1}^{*}, y_{2}^{*}, \ldots, y_{n}^{*}\right)$ is observable if the permuted values all fall within their truncation regions, that is if

$$
y_{i}^{*} \in R_{i} \quad \text { for } \quad i=1,2, \ldots, n
$$




\begin{tabular}{ccc}
$z_{i}$ & $y_{i}$ & $R_{i}=\left[u_{i}, v_{i}\right]$ \\
\hline 1 & 0.75 & {$[0.4,2.0]$} \\
2 & 1.25 & {$[0.8,1.8]$} \\
3 & 1.50 & {$[0.0,2.3]$} \\
4 & 1.05 & {$[0.3,1.4]$} \\
5 & 2.40 & {$[1.1,3.0]$} \\
6 & 2.50 & {$[2.3,3.4]$} \\
7 & 2.25 & {$[1.3,2.6]$}
\end{tabular}

Table 2.3: Table 1: An artificial example of truncated data involving $n=7$ data points.

In figure 1 , and Table 2 , we see that $\left(y_{3}, y_{2}, y_{1}, y_{4}, y_{5}, y_{6}, y_{7}\right)$ is observable, but not $\left(y_{2}, y_{1}, y_{3}, y_{4}, y_{5}, y_{6}, y_{7}\right)$. We define

$$
\mathcal{Y}=\text { set of observable permutations. }
$$

It turns out that $\mathcal{Y}$ has 78 members in the seven-point example.

Permutation tests of the independence hypothesis $H_{o}$ are based on the conditional distribution of $\mathbf{y}^{*}$ in $\mathcal{Y}$ given the ordered values $y_{(1)}, y_{(2)}, \ldots, y_{(n)}$ of the observed response vector $\mathbf{y}$, (assuming for convenience no ties), say

$$
\mathbf{y}_{()}=\left(y_{(1)}, y_{(2)}, \ldots, y_{(n)}\right)
$$

and also given the $z_{i}$ and $R_{i}$ values,

$$
\mathbf{z}=\left(z_{1}, z_{2}, \ldots, z_{n}\right) \quad \text { and } \quad \mathbf{R}=\left(R_{1}, R_{2}, \ldots, R_{n}\right)
$$

Independence Lemma Suppose that $H_{o}$ is true. Then the conditional distribution of $\mathbf{y}^{*}$ given $\mathbf{y}_{()}, \mathbf{z}$, and $\mathbf{R}$ is uniform over $\mathcal{Y}$.

Proof According to $(2.2),(2.3)$, an observable vector $\mathbf{y}^{*}=\left(y_{1}^{*}, y_{2}^{*}, \ldots, y_{n}^{*}\right)$ has $H_{o}$ density 
fig2. 7-point example, showing mle from qself3

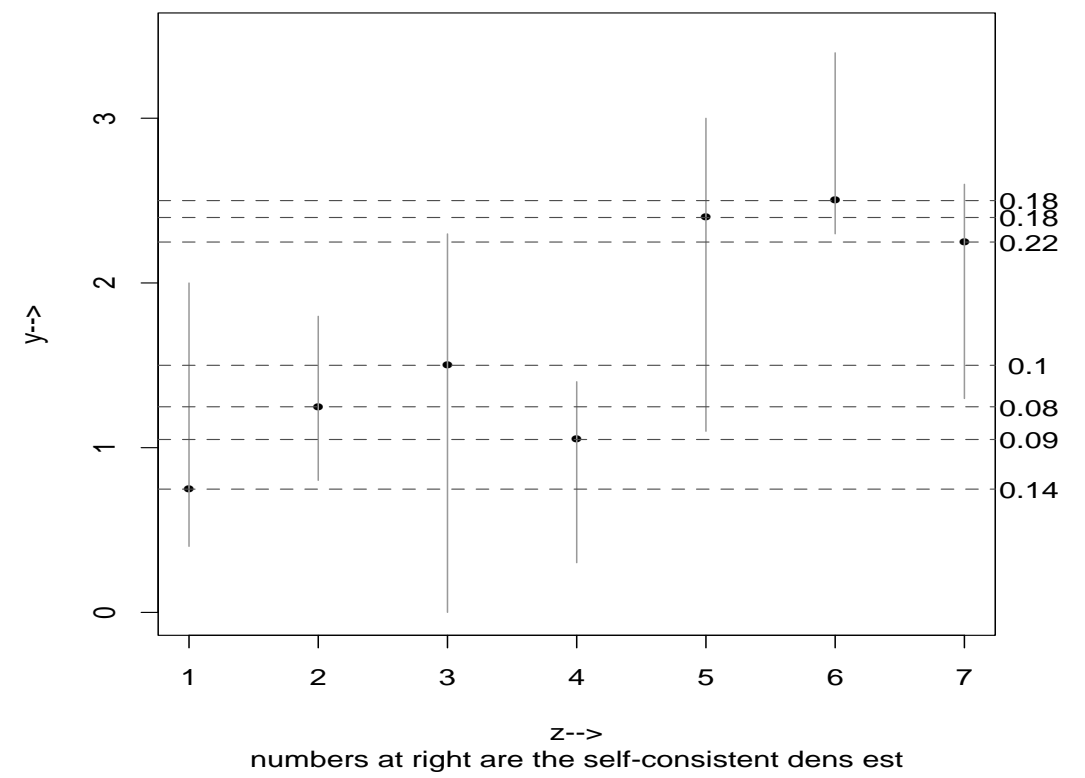

Figure 2: The seven-point example from Table 1; dots indicate points $\left(z_{i}, y_{i}\right)$; vertical bars show truncation regions $R_{i}=\left[u_{i}, v_{i}\right]$; dashed horizontal lines indicate the ordered $y$ values. Numbers at right give the nonparametric MLE for the $y$ distribution assuming that the independence hypothesis $H_{o}$ is true, as explained in Section 4. 


$$
\prod_{i=1}^{n}\left[f\left(y_{i}^{*}\right) / F_{i}\right]=\prod_{i=1}^{n} f\left(y_{i}^{*}\right) / \prod_{i=1}^{n} F_{i}
$$

If $\mathbf{y}^{*} \in \mathcal{Y}$ then $(2.8)$ equals $\prod_{i=1}^{n} f\left(y_{(i)}\right) / \prod_{i=1}^{n} F_{i}$. This has the same value for all $\mathbf{y}^{*} \in \mathcal{Y}$, which proves the lemma.

Permutation tests of $H_{o}$ are carried out in the usual way: we choose a test statistic $S(\mathbf{y})$, with larger values of $S$ indicating stronger disagreement with $H_{o}$, and compare the observed value $s=S(\mathbf{y})$ with the set of permutation values

$$
\mathcal{S}=\left\{S\left(\mathbf{y}^{*}\right), \mathbf{y}^{*} \in \mathcal{Y}\right\}
$$

The p-value of the test is the proportion of $\mathcal{S}$ exceeding $s$.

The tau test. A particular choice of the test statistic $S(\mathbf{y})$ was advocated in Tsai (1990) and Efron and Petrosian $(1992,1994)$, in the context of one-sided truncation. A pair of indices $(i, j)$ is said to be comparable if $y_{i} \in R_{j}$ and $y_{j} \in R_{i}$. Define

$$
\mathcal{C}=\text { set of comparable pairs }
$$

and

$$
\widetilde{\tau}=\sum_{(i, j) \in \mathcal{C}} \operatorname{sign}\left[\left(y_{i}-y_{j}\right)\left(z_{i}-z_{j}\right)\right] / \# \mathcal{C} .
$$

For untruncated data $\# \mathcal{C}=\left(\begin{array}{l}n \\ 2\end{array}\right)$ and $\widetilde{\tau}$ is Kendall's tau statistic. If we decide that the independence hypothesis $H_{o}$ is false then $\widetilde{\tau}$ provides a convenient nonparametric measure of correlation between $y$ and $z$, see Section 3 of Efron and Petrosian (1994); $\widetilde{\tau}=.429$ for the seven-point example.

Here we will use just the numerator of (2.11), 


$$
\widehat{\tau}=\sum_{(i, j) \in \mathcal{C}} \operatorname{sign}\left[\left(y_{i}-y_{j}\right)\left(z_{i}-z_{j}\right)\right]
$$

as the statistic $S(\mathbf{y})$ for testing $H_{o}$, calling this the tau test. For the seven-point example $\widehat{\tau}=3$ and the 78 members of $\mathcal{Y}$ have this distribution of $\widehat{\tau}^{*}$ values,

$$
\begin{array}{cccc}
\widehat{\tau}^{*}: & <3 & =3 & >3 \\
\#: & 63 & 8 & 7
\end{array}
$$

The one-sided p-value for testing $H_{o}$ is $(7+8 / 2) / 78$ (splitting the probability atom at $\widehat{\tau}^{*}=\widehat{\tau}$.)

Permutation tests based on $\widetilde{\tau}$ or $\widehat{\tau}$ are identical for one-sided truncation, since $\# \mathcal{C}$ is the same for all observable permutations, but they can differ under two-sided truncation. The two test statistics give almost the same results for the quasar data. Section 6 also discusses other choices of $S(\mathbf{y})$ that have greater testing power.

\section{Approximate P-Values}

The p-value for the seven-point example was found by complete enumeration of the tau statistic over the set of observable permutations $\mathcal{Y}$. This becomes impossible for data sets

much larger than $n=7$, and we need convenient approximations in order to carry out the test.

It seems like a good approximation should be easy to find. The tau statistic (2.12) has permutation expectation zero,

$$
E_{\text {perm }}\left\{\widehat{\tau}^{*}\right\}=0
$$


since interchanging any comparable pair of indices reverses the sign of the corresponding component of $\widehat{\tau}((3.1)$ is not true for $\widetilde{\tau},(2.11))$. Moreover the components of $\widehat{\tau}$ are shorttailed so that the normal approximation

$$
\widehat{\tau} \sim N\left(0, \sigma_{\text {perm }}^{2}\right)
$$

is likely to have good accuracy, see Theorem 2 of Tsai (1990). However, there seems to be no convenient formula for $\sigma_{\text {perm }}^{2}$, the permutation variance, at least not in the doubly truncated case. A formula does exist when the truncation is only one-sided, as discussed below.

We can use Markov Chain Monte Carlo (MCMC) methods to approximate $\sigma_{\text {perm }}^{2}$. That is we can generate a random walk on $\mathcal{Y}$ that eventually produces uniformly distributed permutation vectors $\mathbf{y}^{*}$, and then estimate $\sigma_{\text {perm }}^{2}$ by the empirical variance of $\widehat{\tau}\left(\mathbf{y}^{*}\right)$. A particularly simple MCMC scheme starts at $\mathbf{y}^{*}=\mathbf{y}$ and proceeds as follows: (1) choose a pair of indices $i, j$ at random; and (2) interchange the $i^{\text {th }}$ and $j^{\text {th }}$ components of $\mathbf{y}^{*}$ if the resulting vector is in $\mathcal{Y}$ (that is if $y_{i}^{*} \in R_{j}$ and $y_{j}^{*} \in R_{i}$ ) otherwise keeping $\mathbf{y}^{*}$ the same.

Elementary Markov Chain Theory says that $\mathbf{y}^{*}$ has its stationary distribution uniform on $\mathcal{Y}$, see Gelman and Rubin (1992). This assumes that $\mathcal{Y}$ is connected by coordinate interchanges, a fact proved recently by Persi Diaconis and Ronald Graham (personal communication). A sufficient number of iterations of steps (1) and (2) make $\mathbf{y}^{*}$ nearly uniformly distributed on $\mathcal{Y}$. Repeating this whole process some large number $B$ of times, perhaps $B=800$, gives independent vectors $\mathbf{y}^{*}(1), \mathbf{y}^{*}(2), \ldots, \mathbf{y}^{*}(B)$, distributed nearly uniformly over $\mathcal{Y}$. Then we can estimate the permutation variance by

$$
\widehat{\sigma}_{\text {perm }}^{2}=\frac{\sum_{b=1}^{B}\left[\widehat{\tau}\left(\mathbf{y}^{*}(b)\right)-\widehat{\tau}(\cdot)\right]^{2}}{B-1} \quad\left[\widehat{\tau}(\cdot)=\sum_{b=1}^{B} \widehat{\tau}\left(\mathbf{y}^{*}(b)\right) / B\right],
$$

and approximate the p-value of the tau test by

$$
\widehat{p}=1-\Phi(\widehat{T}), \quad \widehat{T}=\widehat{\tau}(\mathbf{y}) / \widehat{\sigma}_{\text {perm }}
$$


where $\Phi$ is the standard normal cumulative distribution function (CDF). A more direct approach, but one that tends to require larger values of $B_{1}$ is to estimate $p$ as at (2.9), by

$$
\widehat{p}=\#\left\{\widehat{\tau}^{*}>\widehat{\tau}\right\} / B
$$

An MCMC analysis of the quasar data of Figure 1 was carried out by Duncan Murdoch, personal communication. The number of iterations required to compute $\widehat{T}(0)=\widehat{\tau} / \widehat{\sigma}_{\text {perm }}$ with the same acuracy achieved in Figure 5 was, very roughly, 4,000,000. In any given situation it is hard to know how many iterations of steps (1) and (2) are required to make $\mathbf{y}^{*}$ sufficiently uniform on $\mathcal{Y}$. Generating independent replicates $\mathbf{y}^{*}(b)$ as in (3.3), which is very convenient for error analyses, may be quite inefficient in the MCMC context. An information reference on these difficulties and their possible remedies is Gilks et al. (1993) and the ensuing discussion.

Section 5 discusses a bootstrap approximation for $\widehat{\sigma}_{\text {perm }}^{2}$ that sacrifices the theoretical exactness of the MCMC algorithm for more efficient and more definite numerical results. Bootstrap estimates are used in section 6's analysis of the quasar data. The bootstrap approach has the advantage of applying to any kind of truncated and/or censored data, as mentioned at the end of Section 5 .

Neither MCMC nor the bootstrap are needed in the case of one-sided truncation where the observational regions in (2.11) are all of the form $R_{i}=\left[u_{i}, \infty\right)$. In this case there is a simple way to generate vectors $\mathbf{y}^{*}$ uniform on $\mathcal{Y}$. Define the risk-set numbers

$$
N_{j}=\#\left\{i: u_{i} \leq y_{(j)} \quad \text { and } \quad y_{i} \geq y_{(j)}\right\}
$$

$N_{j}$ is the size of the $j^{\text {th }}$ risk set in the following sense: Looking at figure 2, but with all the regions $R_{i}$ now extending up to infinity, we begin with $y_{(1)}$, the smallest $y$ value, and work upwards. At each $y_{(j)}$ there are $N_{j}$ observable choices of " $z_{i}$ " to go with $y_{(j)}$, all of which are equally likely under $H_{o}$. These are the values of $z$ "at risk" for pairing with $y_{(j)}$. 
For example, there are $N_{2}=3$ possible choices of $z$ to go with $y_{(2)}$, namely 2,3 , or 4 , the actual choice in figure 2 being $z=4$. Altogether there are $N=\prod_{i=1}^{n} N_{i}$ members of $\mathcal{Y}$, $N=324 \quad(=3 \cdot 3 \cdot 3 \cdot 3 \cdot 2 \cdot 2 \cdot 1)$ for the seven-point example.

A uniform choice of $\mathbf{y}^{*}$ in $\mathcal{Y}$ is accomplished by choosing $z_{i}$ uniformly from the $N_{i}$ possible choices at each $y_{(j)}$. this makes it easy to simulate the permutation distribution for any test statistic $S(\mathbf{y})$. The permutation variance of the tau statistic (2.12) turns out to be

$$
\sigma_{\text {perm }}^{2}=4 \sum_{i=1}^{n} V_{i} \quad \text { where } \quad V_{i}=\frac{N_{i}^{2}-1}{12}
$$

as shown for example in Section 3 of Efron and Petrosian (1994). We will use Formula (3.7) in Section 5 to validate the accuracy of the bootstrap estimate of $\sigma_{\text {perm }}^{2}$.

\section{Estimating The Response Distribution}

Question 2 of the introduction asks us to estimate the distribution of the response variable $y$ assuming that the independence hypothesis $H_{o}$ is true. More precisely, we want to estimate the response density $f(y)$ in $(2.2),(2.3)$. This section discusses nonparametric and parametric estimates of $f(y)$ when the data is doubly truncated.

The nonparametric MLE is a discrete distribution putting all of its probability on the observed responses $y_{1}, y_{2}, \ldots, y_{n}$, Turnbull (1976). Let $\mathbf{f}=\left(f_{1}, f_{2}, \ldots, f_{n}\right)$ be a distribution putting probability $f_{i}$ on $y_{i}$, and let $\mathbf{F}=\left(F_{1}, F_{2}, \ldots, F_{n}\right)$ be the vector of observational probabilities $F_{i}=\operatorname{prob}_{\mathbf{f}}\left\{y \in R_{i}\right\}$, so

$$
\mathbf{F}=\mathbf{J f}
$$

where $\mathbf{J}$ is the $n \times n$ matrix describing which $y$ values are included in the regions $R_{i}$, 


$$
J_{i j}= \begin{cases}1 & \text { if } y_{j} \in R_{i} \\ 0 & \text { if } y_{j} \notin R_{i}\end{cases}
$$

According to (2.2), the log likelihood of the observed sample is

$$
\ell=\log \prod_{i=1}^{n}\left(f_{i} / F_{i}\right)
$$

Differentiating (4.3) with respect to $f_{i}$, and using (4.1), gives

$$
\frac{\partial \ell}{\partial f_{i}}=\frac{1}{f_{i}}-\sum_{j=1}^{n} J_{j i} \frac{1}{F_{j}} .
$$

The maximum likelihood equations $\partial \ell / \partial f_{i}=0$ can be expressed as

$$
\frac{1}{\mathbf{f}}=\mathbf{J}^{\prime} \frac{1}{\mathbf{F}}
$$

where $\frac{\mathbf{1}}{\mathbf{f}}=\left(\frac{1}{f_{1}}, \frac{1}{f_{2}}, \ldots, \frac{1}{f_{n}}\right)$ and $\frac{\mathbf{1}}{\mathbf{F}}=\left(\frac{1}{F_{1}}, \frac{1}{F_{2}}, \ldots, \frac{1}{F_{n}}\right)$. Notice that $\ell$ in (4.3) stays the same if $\mathbf{f}$, and hence $\mathbf{F}$, is multiplied by any positive constant, allowing us to ignore the constraint $\sum_{i=1}^{n} f_{i}=1$ in the derivation of (4.5).

Equation (4.5) is the same as Turnbull's self-consistency criterion. We can solve for the MLE $\widehat{\mathbf{f}}$ by beginning with any initial estimate and then iterating between (4.1) and (4.5) (remembering to re-scale after each application of (4.5) so that the estimate of $\mathbf{f}$ sums to 1). The nonparametric MLE $\widehat{\mathbf{f}}$ for the seven-point example is shown at the right edge of Figure 2. The substantial differences between $\widehat{\mathbf{f}}$ and the untruncated MLE $(.14, .14, \ldots, .14)$ are not intuitively obvious from the truncation pattern.

The method just described is an EM algorithm, and often converges quite slowly to the MLE. An alternative algorithm is based on Lynden-Bell's 1971 method for computing the MLE in the case of one-sided truncation. For notational convenience suppose that the $y$ values are indexed in increasing order, so $y_{i}=y_{(i)}$, where we continue to assume that 
there are no ties. Corresponding to density function $\mathbf{f}=\left(f_{1}, f_{2}, \ldots, f_{n}\right)$, the survival curve $\mathbf{G}=\left(G_{1}, G_{2}, \ldots, G_{n}\right)$ and the hazard function $\mathbf{h}=\left(h_{1}, h_{2}, \ldots, h_{n}\right)$ are defined by

$$
G_{j}=\sum_{i \geq j} f_{i} \quad \text { and } \quad h_{j}=f_{j} / G_{j}
$$

We can recover $\mathbf{G}$ and $\mathbf{f}$ from $\mathbf{h}$ via the relationships

$$
G_{j}=\exp \left\{\sum_{i<j} \log \left(1-h_{i}\right)\right\} \quad \text { and } \quad f_{j}=G_{j}-G_{j+1}
$$

Here $G_{1}=G\left(y_{(1)}\right)=1$ by definition.

The following theorem is verified in the Appendix.

Hazard Rate Theorem The nonparametric MLE $\widehat{\mathbf{f}}$ has hazard function $\widehat{\mathbf{h}}$ satisfying

$$
\frac{1}{\widehat{h}_{j}}=N_{j}+\sum_{i=1}^{n} J_{i j} \widehat{Q}_{i}
$$

where $N_{j}$ are the risk-set numbers (3.5), $\left\{J_{i j}\right\}$ are the inclusion indicators (4.2), and

$$
\widehat{Q}_{i}=\widehat{G}_{v_{i}+} / \widehat{F}_{i}
$$

Here $\widehat{\mathbf{F}}=\mathbf{J} \widehat{\mathbf{f}}$ as in (4.1), and $\widehat{G}_{v_{i}+}=\sum\left\{\widehat{f}_{k}: y_{k}>v_{i}\right\}$.

The numerator of $\widehat{Q}_{i}$ is the MLE probability of exceeding $v_{i}$, the upper observational limit for $y_{i}$. In the one-sided truncation case $\widehat{Q}_{i}=0$ since $v_{i}=\infty$, so equation (4.8) takes the form

$$
\frac{1}{\widehat{h}_{j}}=N_{j},
$$

which is Lynden-Bell's (1971) estimate. In this case (4.7) gives the MLE $\widehat{\mathbf{f}}$ directly, without iteration. In the case of two-sided truncation we can begin with (4.10) and iterate (4.7), 
(4.8) to obtain the MLE. This converges quickly if the upper truncation is not severe, as turns out to be the situation for the quasar data.

The solid curve in Figure 3 shows $\log \widehat{G}(y)$, the nonparametric MLE of $\log \left(\sum_{y_{i} \geq y} f_{i}\right)$, for the 210 quasars. The log luminosity $y$ is not the same quantity plotted in figure 1, but is an adjusted version as explained in Section 6. Also shown is the Lynden-Bell estimate (4.10), dashed line, which ignores upper truncation. The two estimates are almost the same so in this case upper truncation has little effect. The MLE algorithm based on (4.7)-(4.8) converges very quickly here.

It is not an accident that the Lynden-Bell estimate of survival is everywhere less than the MLE.

Corollary The Lynden-Bell estimated survival curve, which ignores upper truncation, is less than or equal to the nonparametric MLE.

The proof of the corollary is immediate from a comparison of (4.8) with (4.10), which shows that the estimated hazard rate can only be greater in the Lynden-Bell case.

The curve labeled "SEF" in Figure 3 is derived from a special exponential family in the terminology of Efron and Tibshirani (1996). In this case the SEF density estimate $\widehat{f}(y)$ is the MLE among densities of the form

$$
\log f(y)=\beta_{0}+\beta_{1} y+\beta_{2} y^{2}+\beta_{3} y^{3}
$$

for $y$ in the range of the observed $y_{i}$ values. That is, it maximizes $\prod_{i=1}^{n}\left(f\left(y_{i}\right) / F_{i}\right)$ in $(2.2)$, (2.3) among all choices of $\left(\beta_{1}, \beta_{2}, \beta_{3}\right)$ (with $\beta_{0}$ then determined by the requirement that $f(y)$ integrate to 1 over the interval $\left.\left[y_{(1)}, y_{(n)}\right]\right)$. The appendix describes the calculation of $\widehat{f}(y)$.

Lynden-Bell's estimate and the parametric MLE can behave erratically near the extremes of the $y$ range, where the risk set numbers $N_{j}$ may be small. At the arrowed point in figure 3 for example $N_{1}=2$, giving Lynden-Bell estimates $\widehat{h}_{1}=.50$ and $\widehat{G}\left(y_{(2)}\right)=.50$ according to (4.10) and (4.7). The nonparametric MLE has $\widehat{G}\left(y_{(2)}\right)=.51$. The SEF estimate smooths 
fig3

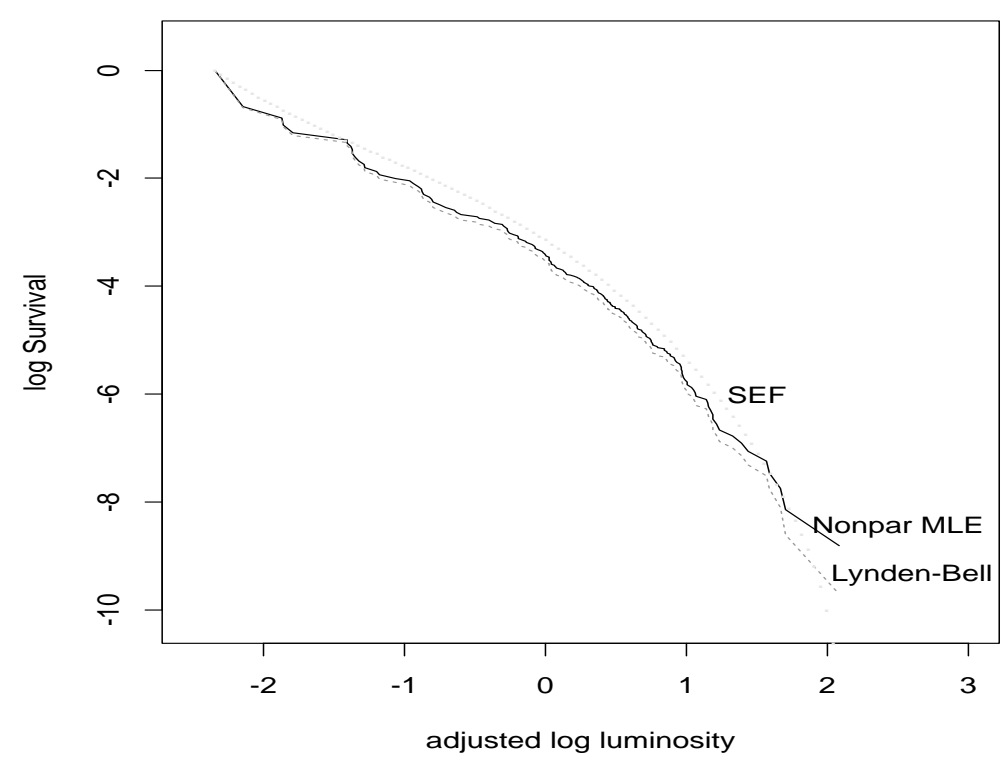

Figure 3: Estimated $\log$ survival curve $\log G(y)$ as a function of the adjusted log luminosity evolution $y_{j}$ for the quasar data, adjusted for luminosity evolution: $\theta=2$, as explained in Section 6; solid curve is nonparametric MLE; dashed curve is Lynden-Bell estimate (4.10), ignoring upper truncation; smooth dotted curve is cubic special exponential family. 
out the bumps in the nonparametric MLE, here giving $\widehat{G}\left(y_{(2)}\right)=.72$.

SEF estimates are less variable than the nonparametric MLE, but can be biased if based upon an incorrect model. The cubic model in Figure 3 was chosen by successive significance tests, as explained in the appendix. A small simulation study showed that percentile points of the cubic SEF estimates had roughly half the standard deviation of their nonparametric MLE counterparts.

\section{Bootstrap Tests for Independence}

We now return to the question of testing the independence hypothesis $H_{o},(2.2)$. this section discusses bootstrap approximations to the permutation p-values of Section 2. Most of the discussion is in terms of the tau test, but the method applies to any test statistic as mentioned at section's end, and can be extended to arbitrarily complicated patterns of data censoring and truncation.

Let $\widehat{f}(y)$ be an estimate of the density for the response variable $y$ calculated as in Section 4 , assuming that $H_{o}$ is true. The estimate $\widehat{f}$ might be the nonparametric MLE or an SEF

estimate as in Figure 3. We can use $\widehat{f}$ to draw a bootstrap sample $\mathbf{y}^{*}$ by following the recipe in $(2.2),(2.3): \mathbf{y}^{*}=\left(y_{1}^{*}, y_{2}^{*}, \ldots, y_{n}^{*}\right)$ has independent components, with the $i^{\text {th }}$ component's density being

$$
\left.\begin{array}{cl}
\widehat{f}\left(y_{i}^{*}\right) / \widehat{F}_{i} & \text { for } y_{i}^{*} \in R_{i} \\
0 & \text { for } y_{i}^{*} \notin R_{i}
\end{array}\right\} \text { independently } i=1,2, \ldots, n
$$

where $\widehat{F}_{i}=\int_{R_{i}} \widehat{f}(y) d y$.

An approximate version of the tau test can be carried out by generating $B$ independent bootstrap samples $\mathbf{y}^{*}$ and then proceeding as in (3.3), (3.4) to get the approximate p-value $\widehat{p}=1-\Phi(\widehat{T})$. How big need $B$ be? Letting 


$$
T=\widehat{\tau}(\mathbf{y}) / \sigma_{\text {perm }} \text { and } \widehat{T}=\widehat{\tau}(\mathbf{y}) / \widehat{\sigma}_{\text {perm }}
$$

standard normal-theory calculations show that

$$
s d_{*}\{\widehat{T} / T\} \doteq 1 / \sqrt{2 B}
$$

where $s d_{*}$ indicates the simulation standard deviation. This gives

$\begin{array}{cccccc}\mathrm{B}: & 50 & 100 & 200 & 400 & 800 \\ s d_{*} & 0.10 & .07 & .05 & .035 & .025\end{array}$

so we need $B=800$ bootstrap replications to determine $\widehat{T}$ within about $2.5 \%$ of its ideal value T. These same calculations apply to the MCMC approach in (3.3), (3.4). $B=800$ is large enough to permit a check in the normality assumption leading to the estimate $\widehat{p}=1-\Phi(\widehat{T})$, and a retreat to the nonparametric estimate (3.5) if normality looks dubious.

As a check on the bootstrap test, $\widehat{\sigma}_{\text {perm }}$ was estimated using $B=800$ replications for the data in Figure 1, but ignoring the upper bounds of the truncation regions (i.e. taking all $\left.v_{i}=\infty\right)$. The estimate was $\widehat{\sigma}_{\text {perm }}=.0441 \pm .0011$, " \pm " indicating the bootstrap simulation error, agreeing nicely with the exact permutation standard deviation $\sigma_{\text {perm }}=.0445$ obtained from (3.7).

The bootstrap approximation for $\sigma_{\text {perm }}$ looks much different than the MCMC approach of Section 3. In particular it requires a preliminary estimate of $f(y)$. The brief discussion in the appendix shows that the bootstrap and permutation calculations are more alike than they appear, and that similar approximations are used in more familiar statistical contexts.

There is nothing special about the tau statistic as far as bootstrap or permutation methods are concerned. Section 6 mentions a more powerful version of tau that puts greater weight on those terms in (2.11) having bigger values of $\left|z_{i}-z_{j}\right|$. We could in fact employ 
any test statistic $S(\mathbf{y})$, and then use the bootstrap or MCMC to approximate the comparison set $\mathcal{S}$ and p-value in (2.9). The main advantage of tau-like test statistics is that they have bootstrap or permutation expectations zero under $H_{o}$, and nearly normal distributions, which eases the task of finding p-values by simulation.

Tsai (1990, Section 4) shows that the tau test of independence can be applied to data that is truncated below and censored above. In principle, bootstrap tests can be applied to any form of truncated and/or censored data. The first step is to estimate the response density $f(y)$ using Turnbull's (1976) nonparametric self-consistency algorithm. Then bootstrap samples $\mathbf{y}^{*}$ are drawn as in (5.1), taking into account each $y_{i}$ 's pattern of truncation and censoring, and the p-value approximated by comparing the test statistic $S(\mathbf{y})$ with $\mathcal{S}=\left\{S\left(\mathbf{y}^{*}(1)\right), S\left(\mathbf{y}^{*}(2)\right), \ldots, S\left(\mathbf{y}^{*}(B)\right)\right\}$. Romano (1988) gives a general discussion, and validation, of this kind of "null hypothesis bootstrap" test procedure.

\section{The Quasar Data}

Our estimation and testing theory will now be applied to the quasar data of Figure 1. First the situation will be described more carefully. The original dataset consisted of independently collected quadruplets

$$
\left(z_{i}, m_{i}, a_{i}, b_{i}\right) \quad i=1,2, \ldots, n
$$

where $z_{i}$ is the redshift of the $i^{\text {th }}$ quasar and $m_{i}$ is its apparent magnitude. The numbers $a_{i}$ and $b_{i}$ are lower and upper truncation limits on $m_{i}$. Quasars with apparent magnitude above $b_{i}$ were too dim to yield dependable redshifts (remembering that bigger values of $m$ correspond to dimmer objects.) The lower limit $a_{i}$ was used to avoid confusion with nonquasar steller objects. In this study $a_{i}=16.08$ for all $i$, while $b_{i}$ varied between 18.494 and 
18.934. The full dataset comprised $n=1052$ quasars. Here we are considering a randomly selected subset of $n=210$ quadruplets (6.1).

Farther quasars appear dimmer of course, that is they tend to have bigger values of $m_{i}$. Hubble's law, which says that distance is proportional to redshift, allows us to transform apparent magnitudes into a luminosity measurement that should be independent of distance. This transformation depends on the cosmological model assumed. The log luminosity values $y_{i}$ in Figure 1 were obtained from a formula $y_{i}=t\left(z_{i}, m_{i}\right)$ that takes into account relativistic effects of the distance,

$$
y_{i}=19.894-2.303 \frac{m_{i}}{2.5}+2 \log \left(Z_{i}-Z_{i}^{1 / 2}\right)-\frac{1}{2} \log \left(Z_{i}\right),
$$

where $Z_{i}=1+z_{i}$. Formula (6.2) is derived from the Einstein-deSitter cosmological model, Weinberg (1972). The last term makes the so-called K-correction, taking into account the shifting of the spectrum due to redshift.

Larger values of $y_{i}$ correspond to intrinsically brighter quasars in Figure 1. The truncation limits $R_{i}=\left[u_{i}, v_{i}\right]$ were obtained by applying transformation (6.2) to the observational limits for $m_{i}$,

$$
u_{i}=t\left(z_{i}, b_{i}\right) \quad \text { and } \quad v_{i}=t\left(z_{i}, a_{i}\right)
$$

This makes $u_{i}$ and $v_{i}$, the lower and upper dashes in figure 1, strongly increasing functions of $z_{i}$, even though $a_{i}$ and $b_{i}$ are not.

One of the principal goals of the quasar investigations is to study luminosity evolution: quasars may have been intrinsically brighter in the early universe and evolved toward a dimmer state as time went on. This would tend to make the points on the right side of Figure 1 higher since larger redshifts correspond to quasars seen longer ago. However in the absence of luminosity evolution we should have $y_{i}$ independent of $z_{i}$ except for truncation effects. This brings us back to Question 1 of the introduction. Testing the independence 
hypothesis $H_{o}$ amounts to testing for the absence of luminosity evolution.

A convenient one-parameter model for luminosity evolution says that the expected log luminosity increases linearly as $\theta \cdot \log (1+z)$, with $\theta=0$ corresponding to no evolution. If $\theta$ is a hypothesized value of the evolution parameter then instead of $y_{i}$ being independent of $z_{i}$ we should test the null hypothesis " $H_{\theta}$ ",

$$
H_{\theta}: \quad y_{i}(\theta)=y_{i}-\theta \cdot \log \left(1+z_{i}\right) \quad \text { independent of } \quad z_{i}
$$

Figure 4 shows plots of the data for $\theta$ equal 0,2 , and 4 , all for our same set of 210 quasars. Notice that the truncation regions $R_{i}=\left(u_{i}, v_{i}\right)$ also change with $\theta$,

$$
u_{i}(\theta)=u_{i}-\theta \cdot \log \left(1+z_{i}\right) \quad \text { and } \quad v_{i}(\theta)=v_{i}-\theta \cdot \log \left(1+z_{i}\right)
$$

We can apply the tau test to each of the null hypotheses $H_{\theta}$. This was done for values of $\theta$ between 0 and 4 with the results shown in figure 5 . The solid curve is the standardized test statistic $\widehat{T}=\widehat{\tau} / \widehat{\sigma}_{\text {perm }}$, (3.4), with $\widehat{\sigma}_{\text {perm }}$ determined by bootstrap sampling. $B=800$ bootstrap replications (5.1) were drawn for $\theta=0, .5,1, \ldots, 4$. Almost exactly the same curve was obtained using $\widetilde{\tau},,(2.11)$, in place of $\widehat{\tau}$.

We see that $\widehat{T}(0)=2.20$, giving an approximate one-sided p-value $1-\Phi(2.13)=.015$. The tau test rejects the null hypothesis of independence $H_{o}$ in favor of a positive value of the luminosity parameter $\theta$. At $\widehat{\theta}=2.38$ we have $\widehat{T}(\widehat{\theta})=0$. The $\widehat{T}(\theta)$ curve crosses \pm 1.645 at $[1.00,3.20]$, which provides an approximate $90 \%$ central confidence interval for $\theta$. As a point of comparison, using all 1052 quasars gave $\hat{\theta}=2.11$ and $90 \%$ interval $[1.38,2.63]$.

If we are willing to ignore the upper truncation limits (setting all the $v_{i}=\infty$ ) we can employ the more exact one-sided tau test (3.5)-(3.6). These results, which did not involve bootstrap sampling, were only slightly more significant than those for the two-sided test, as shown by the dots in Figure 5.

The choice $\theta=2$ makes the adjusted $\log \operatorname{luminosity} y_{i}(2)=y_{i}-2 \log \left(1+z_{i}\right)$ approximately 

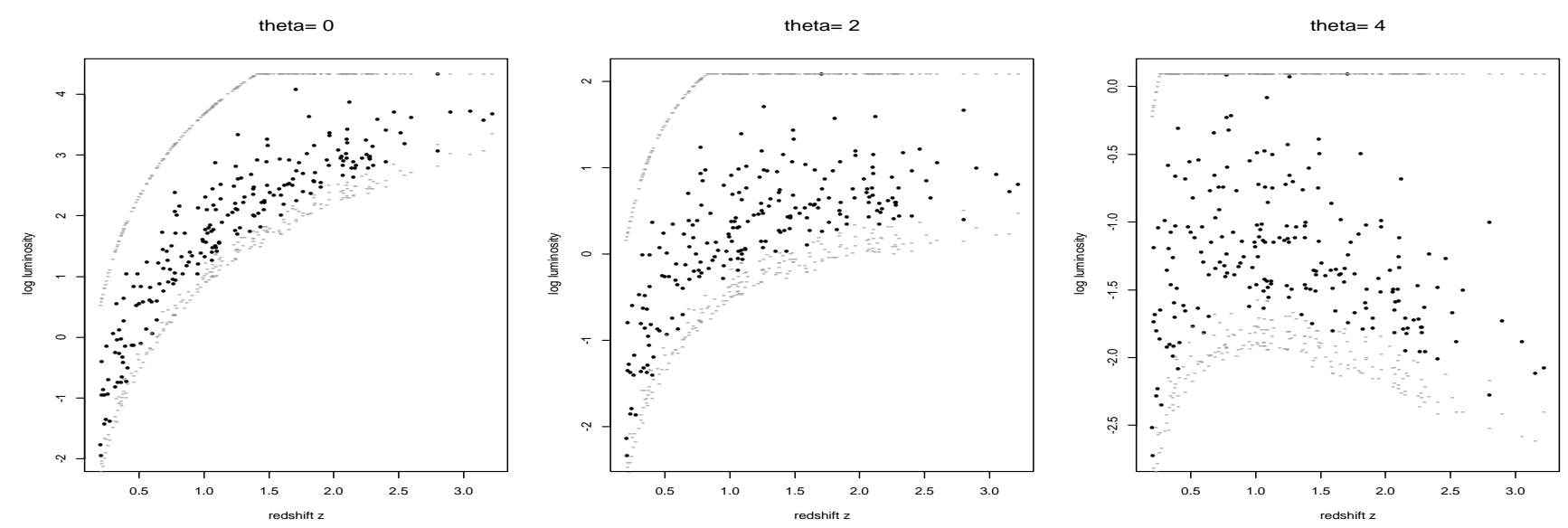

Figure 4: Plots of the quasar data of Figure 1 for three choices of the luminosity evolution parameter $\theta ; \theta=0$ corresponds to Figure 1 ; other values of $\theta$ plot $\left(z_{i}, y_{i}(\theta)\right)$, (6.4), with limits $u_{i}(\theta)$ and $v_{i}(\theta),(6.5)$. 


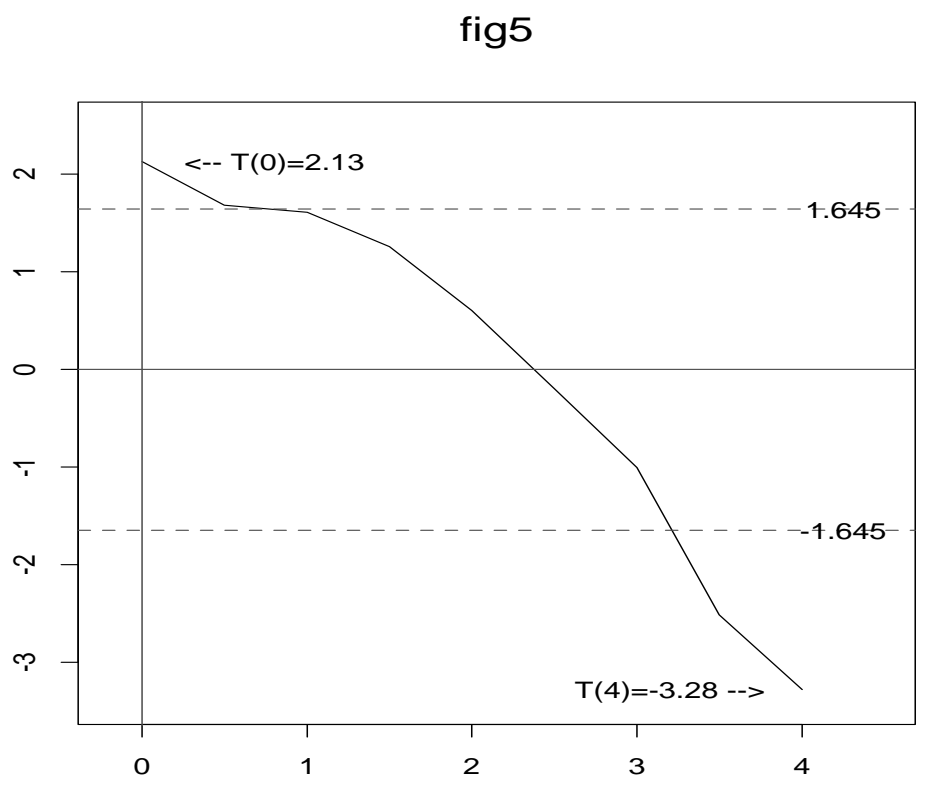

Figure 5: Tau test of the null hypotheses $H_{\theta}$, (6.4), for the 210 quasars; $\theta=0,0.5,1, \ldots, 4$; solid curve $\widehat{T}=\widehat{\tau} / \widehat{\sigma}_{\text {perm }},(3.4)$, crosses zero at $\widehat{\theta}=2.38 ; \widehat{\sigma}_{\text {perm }}$ found by bootstrap sampling (5.1); $90 \%$ central confidence interval $\theta \in[1.00,3.20]$. Dots indicate $\widehat{T}$ values assuming only one-sided truncation. 
independent of $z_{i}$ so we can estimate its density $f(y)$ as in Section 4 . Figure 3 shows the estimated $\log$ survival curve $\log \widehat{G}$. It curves sharply downward for large values of $y$, which is a fortunate thing for the testing results. If $\log \widehat{G}$ were linear then $f(y)$ would correspond to a one-sided exponential density. Because of the exponential's memoryless property it is impossible to test for independence in the exponential case (unless the lower endpoint of the exponential density exceeds some of the lower truncation limits $u_{i}$ ).

On the other hand, the MLE $\widehat{f}(y)$ consistently estimates an exponential tail even if $y_{i}$ and $z_{i}$ are not independent. It is a good idea to estimate $f(y)$ or $G(y)$ even if Question 1 is of primary interest. Should the MLE turn out to be exponential then any testing procedure will be futile.

Model (6.4) gives us reason to question the efficacy of the tau statistic in this situation. Let $\theta_{o}$ be the true value of the evolution parameter. Then the difference of two of the luminosity measurements in Figure 1 , where $\theta=0$, can be expressed as

$$
y_{i}-y_{j}=y_{i}\left(\theta_{o}\right)-y_{j}\left(\theta_{o}\right)+\theta_{o}\left(w_{i}-w_{j}\right) \quad\left[w_{i}=\log \left(1+z_{i}\right)\right]
$$

The differences $y_{i}\left(\theta_{o}\right)-y_{j}\left(\theta_{o}\right)$ are symmetrically distributed about zero, except for truncation effects so (6.6) suggests that $(i, j)$ pairs with bigger values of $\left|w_{i}-w_{j}\right|$ will be more informative in testing for deviations from $H_{o}$. An analysis of the 4907 comparable pairs for the data in Figure 1 verified that those with large values of $\left|w_{i}-w_{j}\right|$ were contributing more consistently to the tau statistics:

$$
\begin{array}{cccccc}
\left|w_{i}-w_{j}\right|: & 0 & .25 & .5 & .75 & 1 \\
\operatorname{prob}\left\{\operatorname{sign}\left(y_{i}-y_{j}\right)\left(z_{i}-z_{j}\right)=1\right\} & .51 & .56 & .59 & .62 & .63
\end{array}
$$

A modified version of the tau statistic (2.11) was tried on the quasar data,

$$
\tilde{\tau}^{\prime}=\sum_{\mathcal{C}}\left|\log \left(w_{i} / w_{j}\right)\right| \operatorname{sign}\left[\left(y_{i}-y_{j}\right)\left(z_{i}-z_{j}\right)\right] / \sum_{\mathcal{C}}\left|\log \left(w_{i} / w_{j}\right)\right|
$$


but it showed only modest improvements over either $\widetilde{\tau}$ or $\widehat{\tau}$. The gains were more substantial when all 1052 quasars were included, giving standardized statistic $\tilde{T}^{\prime}=3.21$ compared to $\widehat{T}=2.77$ for testing $H_{o}$. 


\section{Appendix}

\section{A Proof of the hazard rate theorem (4.8)}

Because the nonparametric MLE puts all of its probability on the observed responses, and because it is invariant under monotonic transformation of the $y$ scale, we can assume that $y_{i}=i$ for $i=1,2, \ldots$, and that $R_{i}=\left[u_{i}, v_{i}\right]$ where $u_{i}$ and $v_{i} \in\{1,2, \ldots, n\}$ (again assuming no tied $y$ values.) In addition to $N_{j}=\#\left\{i: u_{i} \leq j\right.$ and $\left.i \geq j\right\}$, (3.5), we define

$$
M_{j}=\#\left\{i: u_{i}=j\right\}
$$

It is easy to see that

$$
M_{1}=N_{1} \quad \text { and } \quad M_{j}=N_{j}-N_{j-1}+1 \quad \text { for } j=2,3, \ldots, n \text {. }
$$

We will also assume that

$$
M_{n}=0
$$

because if this were not true then the largest observation $y_{i}=n$ would have been truncated to the degenerate interval $R_{n}=[n, n]$, and we could reduce the sample size to $n-1$. (A3) implies $N_{n-1}=N_{n}+1=2$ according to (A2), since $N_{n}=1$.

Following the notation of section 4 , a density $\mathbf{f}=\left(f_{1}, f_{2}, \ldots f_{n}\right)$ has likelihood $L=$ $\prod_{i=1}^{n} f_{i} / F_{i}$ for the observed sample. The hazard function $\mathbf{h}=\left(h_{1}, h_{2}, \ldots, h_{n}\right)$ has $h_{n}=1$, so we need only consider its first $n-1$ coordinates. The following lemma expresses the likelihood in terms of the hazard rate.

Lemma The likelihood corresponding to $\left(f_{1}, f_{2}, \ldots, f_{n}\right)$ or $\left(h_{1}, h_{2}, \ldots, h_{n-1}\right)$ is 


$$
L=\prod_{i=1}^{n} \frac{f_{i}}{F_{i}}=\left[\prod_{j=1}^{n-1} h_{j}\left(1-h_{j}\right)^{N_{j}-1}\right] /\left[\prod_{i=1}^{n}\left\{1-\prod_{u_{i}}^{v_{i}}\left(1-h_{k}\right)\right\}\right]
$$

Proof In the one-sided case of no upper truncation, where all the $v_{i}=n$, the denominator in (A4) equals 1 ( since $h_{n}=1$ ), so that (A4) becomes

$$
\prod_{i=1}^{n} \frac{f_{i}}{F_{i}}=\prod_{j=1}^{n-1} h_{j}\left(1-h_{j}\right)^{N_{j}-1}
$$

This is easy to verify, starting from $h_{j}=f_{j} / G_{j}$ :

$$
\begin{aligned}
\prod_{j=1}^{n-1} h_{j}\left(1-h_{j}\right)^{N_{j}-1} & =\prod_{j=1}^{n-1} \frac{f_{j}}{G_{j}}\left(\frac{G_{j+1}}{G_{j}}\right)^{N_{j}-1}=\left[\prod_{j=1}^{n-1} f_{j}\right]\left[\frac{G_{n}^{N_{n-1}-1}}{G_{1}^{N_{1}}, G_{2}^{N_{2}+1} \ldots G_{n-1}^{N_{n-1}-N_{n-2}+1}}\right] \mathrm{A} . \\
& =\prod_{j=1}^{n} f_{j} / \prod_{j=1}^{n-1} G_{j}^{M_{j}}
\end{aligned}
$$

where we have used $G_{n}=f_{n}, \quad N_{n-1}=2$, (A2), and (A3). But in the one-sided case $\prod_{j=1}^{n-1} G_{j}^{M_{j}}=\prod_{i=1}^{n} F_{i}$, since each $F_{i}$ must equal some $G_{j}$, so (A6) proves (A5).

In the two-sided case

$$
\prod_{i=1}^{n} \frac{f_{i}}{F_{i}}=\left[\prod_{i=1}^{n} \frac{f_{i}}{G_{u_{i}}}\right]\left[\prod_{i=1}^{n} \frac{G_{u_{i}}}{F_{i}}\right]
$$

But the first bracketed factor on the right is the same as $\prod_{i=1}^{n}\left(f_{i} / F_{i}\right)$ for the one-sided case, and formula (4.7) says that

$$
\frac{G_{u_{i}}}{F_{i}}=\frac{G_{u_{i}}}{G_{u_{i}}-G_{v_{i}+}}=\left[1-\prod_{u_{i}}^{v_{i}}\left(1-h_{k}\right)\right]^{-1}
$$

which together give the lemma (A4). Finally, differentiating the log likelihood

$$
\log L=\sum_{j=1}^{n-1}\left[\log h_{j}+\left(N_{j}-1\right) \log \left(1-h_{j}\right)\right]-\sum_{i=1}^{n} \log \left[1-\prod_{u_{i}}^{v_{i}}\left(1-h_{k}\right)\right]
$$


gives

$$
\frac{\partial \log L}{\partial h_{j}}=\frac{1}{h_{j}}-\frac{1}{1-h_{j}}\left[\left(N_{j}-1\right)+\sum_{i: j \in R_{i}} \frac{G_{v_{i+}}}{F_{i}}\right],
$$

which is equivalent to the hazard rate theorem (4.8), (4.9).

The likelihood equality (A.5) for the one-sided case can be obtained directly from familiar survival analysis arguments involving successive conditionally independent binomial likelihoods. See section 2 of Efron (1988). In the two-sided case successive conditioning gives the messier expression

$$
L=\left[\prod_{j=1}^{n} \prod_{i \in \mathcal{N}_{j}}\left(1-h_{i j}\right)\right]\left[\prod_{j=1}^{n} \frac{h_{j j}}{1-h_{j j}}\right],
$$

when $h_{i j}$ is the hazard function for $y_{i}$,

$$
h_{i j}=f_{j} / \sum_{j \leq k \leq v_{i}} f_{k}
$$

and $\mathcal{N}_{j}=\left\{i: u_{i} \leq j\right.$ and $\left.i \geq j\right\}$, the " $j^{\text {th }}$ risk set". The lemma says that this reduces to (A4).

Special Exponential Families Classic exponential families such as the normal, Poisson, and binomial have mathematical properties that greatly simplify their use. Modern computational equipment allows us to design special exponential families (SEF) for particular applications without worrying about mathematical tractability. The SEF appearing in Figure 3 has a density of the form

$$
f_{\eta}(y)=e^{\eta^{\prime} t(y)-\phi(\eta)} \quad \text { for } \quad y e \mathcal{Y}
$$

where $t(y)=\left(y, y^{2}, y^{3}\right), \quad \eta=\left(\eta_{1}, \eta_{2}, \eta_{3}\right), \mathcal{R}=\left[\min \left(y_{i}\right), \max \left(y_{i}\right)\right]$ and $\phi(\eta)$ is chosen to make $\int_{\mathcal{Y}} f_{\eta}(y) d y=1$ 
The cubic SEF shown in Figure 3 used the MLE $\widehat{\eta}$ of the parameter vector $\eta$. In order to calculate $\widehat{\eta}$ we define

$$
T_{\eta}(g)=\int_{\mathcal{R}} g(y) e^{\eta^{\prime} t(y)} d y,
$$

where $g(y)$ may be a vector or matrix function of $y$ in which case the integrals in (A.13) are carried out component-wise. Then the score function $\dot{\ell}_{\eta}(\mathbf{y})=\frac{\partial}{\partial \eta} \log f_{\eta}(\mathbf{y})$ for the truncated data structure $(2.2),(2.3)$ is

$$
\dot{\ell}_{\eta}(\mathbf{y})=\sum_{i=1}^{n}\left[t\left(y_{i}\right)-T_{\eta}\left(I_{i} \cdot t\right) / T_{\eta}\left(I_{i}\right)\right]
$$

where $I_{i}(y)$ is the indicator function for $R_{i}$, as in Efron and Tibshirani (1996). The second derivative matrix is given by

$$
\left.-\ddot{\ell}_{\eta}(\mathbf{y})=\sum_{i=1}^{n}\left\{T_{\eta}\left(I_{i} \cdot t^{2}\right) / T_{\eta}\left(I_{i}\right)-\left[T_{\eta}\left(I_{i} \cdot t\right) / T_{\eta}\left(I_{i}\right)\right)\right]^{2}\right\},
$$

with $t^{2}$ indicating the $n \times n$ outer product matrix $\left(t_{i} t_{j}\right)$.

The MLE $\widehat{\eta}$, satisfying $\dot{\ell}_{\widehat{\eta}}(y)=0$, is found by Newton-Raphson iteration

$$
\widehat{\eta}(k+1)-\widehat{\eta}(k)=\left[-\ddot{\ell}_{\widehat{\eta}(k)}(\mathbf{y})\right]^{-1} \dot{\ell}_{\widehat{\eta}(k)}(\mathbf{y}),
$$

These calculations go quickly because they involve only one-dimensional integrals (A.13), though many of them. Successive models $t(y)=y_{j} t(y)=\left(y, y^{2}\right), \ldots$ were tried on the data for Figure 2. Standard hypothesis tests led to the choice of a cubic model. These tests used the estimated covariance matrix $\left[-\ddot{\ell}_{\widehat{\eta}}(\mathbf{y})\right]^{-1}$ from (A.15) to assess the significance of the $\widehat{\eta}$ coefficients, e.g. whether or not $\widehat{\eta}_{3}$ is significantly non-zero for the cubic model.

Note The nonparametric MLE is itself an SEF estimate, where the components of $t(y)$ in (A.12) are delta functions on the observed $y_{i}$ values, $t(y)=\left(\delta\left(y-y_{1}\right), \delta\left(y-y_{2}\right), \ldots, \delta\left(y-y_{n}\right)\right)$. 
Bootstrap and Permutation Calculations Section 5 discusses bootstrap approximations to permutation tests for the null hypothesis of independence $H_{o}$. The situation there, involving doubly truncated data, looks complicated but in fact it is perfectly analogous to much simpler and more familiar statistical problems.

Consider the problem of testing the equality of two binomial probabilities. We observe independent Bernoulli variates

$$
\begin{aligned}
y_{11}, y_{12}, \ldots y_{1 n_{1}} & \sim B i\left(1, \pi_{1}\right) \\
y_{21}, y_{22}, \ldots y_{2 n_{2}} & \sim B i(1, \pi)
\end{aligned}
$$

and wish to test the null hypothesis $H_{o}$ that $\pi_{1}=\pi_{2}=\pi$ (say). Letting $x_{1}=\sum_{i=1}^{n_{1}} y_{1 i} \quad$ and $\quad x_{2}=$ $\sum_{i=1}^{n_{2}} y_{2 i}$ we can arrange the data in a $2 \times 2$ table, first row $\left(x_{1}, n_{1}-y_{2}\right)$ and second row $\left(x_{2}, n_{2}-x_{2}\right)$, in which case $H_{o}$ is the usual hypothesis of independence for the table.

If $H_{o}$ is true than $x_{+}=x_{1}+x_{2}$ is a sufficient statistic and $x_{+} \sim B i\left(n_{+}, \pi\right)$ with $n_{+}=n_{1}+$ $n_{2}$. Moreover all $n_{+}$! permutations of the combined data vector $\mathbf{y}=\left(y_{11}, y_{12}, \ldots, y_{2 n_{2}}\right)$ are equally likely given $x_{+}$, allowing us to construct permutation tests for $H_{o}$ without worrying about the nuisance parameter $\pi$. The permutation test based on the statistic

$$
S=p_{1}-p_{2} \quad\left[p_{1}=x_{1} / n_{1} \quad p_{2}=x_{2} / n_{2}\right]
$$

is Fisher's exact test for independence. $S$ has permutation expectation zero and variance

$$
\sigma_{\text {perm }}^{2}=\frac{n_{+}}{n_{+}-1} \widehat{\pi}(1-\widehat{\pi})\left(\frac{1}{n_{1}}+\frac{1}{n_{2}}\right), \quad\left[\widehat{\pi}=x_{+} / n_{+}\right]
$$

Suppose we did not know formula (A20) and wished to approximate $\sigma_{\text {perm }}^{2}$ by bootstrap simulation. The null hypothesis bootstrap replaces (A18) with

$$
y_{11}^{*}, y_{12}^{*}, \ldots, y_{2 n_{2}}^{*} \stackrel{\text { ind }}{\sim} \operatorname{Bi}(1, \widehat{\pi})
$$


giving

$$
S^{*}=p_{1}^{*}-p_{2}^{*}=\sum_{i=1}^{n_{1}} y_{1 i}^{*} / n_{1}-\sum_{i=1}^{n_{2}} y_{2 i}^{*} / n_{2} .
$$

$S^{*}$ has bootstrap expectation zero and variance

$$
\widehat{\sigma}_{\text {perm }}^{2}=\widehat{\pi}(1-\widehat{\pi})\left(\frac{1}{n_{1}}+\frac{1}{n_{2}}\right) .
$$

The usual $\chi^{2}$ statistic for testing independence is exactly $S^{2} / \widehat{\sigma}_{\text {perm }}^{2}$.

The bootstrap sampling is unconditional in the sense that the samples do not necessarily have $x_{+}^{*}=x_{+}$. Nevertheless we get an approximation that is accurate to second order,

$$
\widehat{\sigma}_{\text {perm }}^{2} / \sigma_{\text {perm }}^{2}=1+O\left(1 / n_{+}\right) .
$$

It can be shown that this is no accident, and that second-order accuracy follows for any situation of the following sort: the observed data can be written as $(A, B)$ where under $H_{o}, A$ has an exponential family of densities and the conditional density $f(B \mid A)$ does not depend on the parameters of the exponential family.

In the binomial situation $A=x_{+}$and $B$ is a permutation of a vector of $x_{+} 1$ 's and $n_{+}-x_{+}$ 0 's. Under $H_{o}, A \sim B i\left(n_{+}, \pi\right)$ and $B \mid A$ is uniform. It is easiest to see the connection with our truncated data problem through the SEF formulation. Formula (A.13) leads to an exponential family of densities for $\mathbf{y}$ under the truncated data model (2.2), with sufficient statistic $A=\sum_{i=1}^{n} t\left(y_{i}\right)$. As the exponential family (A.13) grows larger, say by including more polynomial terms in $t(y)=\left(y, y^{2}, y^{3}, y^{4}, \ldots\right), A$ itself gets bigger, but we can always take $A=\mathbf{y}_{()}$, the order statistic of $\mathbf{y}$. But $A=\mathbf{y}_{()}$is equivalent to taking $A=\mathcal{Y}$ (since the regions $R_{i}$ are known ancillaries), as we did for the permutation tests of section 2 . 


\section{References}

Efron, B. and Tibshirani R. (1996). "Using specially designed exponential families for density estimation." Annals Stat 24, p. 2431-2461.

Efron, B. (1996). "Empirical Bayes methods for combining likelihoods" (with discussion) Journal of American Statistical Association 91, p. 538-565.

Efron, B. and Petrosian V. (1994), "Survival analysis of the gamma-ray burst data" Journal of American Statistical Association 89, p. 452-462.

Efron, B. and Petrosian V. (1992) "A simple test of independence for truncated data with applications to redshift surveys" The Astrophysical Journal 399, p. 345-352.

Efron, B. (1988) "Logistic regression survival analysis, and the Kaplan-Meier curve" Journal of American Statistical Association 83, p. 414-425.

Gelman, A. and Rubin D. (1992) "Iterative simulation using single and multiple sequences" (with discussion) Statistical Science 7, p. 457-511.

Gilks, W., Clayton D. et al. (1993) "Modeling complexity: applications of Gibbs sampling in medicine" (with discussion) Journal of the Royal Statistical Society B 55, p. 39-102.

Lynden-Bell, D. (1971) "A method of allowing for known observational selection in small samples applied to 3CR quasars" Mon. Nat. R. Ast. Soc. 155, p. 95-118.

McLaren C., Wagstaff M, Brittegram G., Jacobs A. (1991) "Detection of two-component mixtures of lognormal distributions in grouped, doubly truncated data analysis of red blood cell volume distributions" Biometrics 47, p. 607-622.

Romano, J. (1988) "A bootstrap revival of some nonparametric distance tests" Journal of American Statistical Association 83, p. 698-708.

Tsai, W. (1990) "Testing the independence of truncation time and failure time" Biometrika 77, p. $169-77$. 
Turnbull, B. (1996) "The empirical distribution function with arbitrarily grouped censored, and truncated data" Journal of the Royal Statistical Society B38, p. 290-295.

Weinberg, S. (1972) Gravitation and Cosmology, Wiley, New York. 